\title{
ANOMALOUS WEAKLY NONERGODIC BROWNIAN MOTIONS IN NONUNIFORM TEMPERATURES*
}

\author{
ANDRZEJ FulińsKI
}

The M. Smoluchowski Institute of Physics, Jagiellonian University Reymonta 4, 30-059 Kraków, Poland

(Received April 11, 2013)

It is shown that ergodicity breaking and anomalous Brownian motion are not limited to composite and/or disordered systems, but can be generated also in simple fluids or solids with time-dependent and/or spatially nonuniform temperature $T(t, \boldsymbol{r})$. A few examples of simple arrangements easy for experimental realization are discussed in detail. Proposed measurements will enable also the observation of transitions from ergodic to weakly nonergodic and from normal to anomalous diffusion. Similar behaviour can be expected in inflationary systems with time-dependent metric, and in expanding gases.

DOI:10.5506/APhysPolB.44.1137

PACS numbers: 05.40.Jc, 02.50.Ey, 05.45.Tp

\section{Introduction}

Ergodic theory is a cornerstone of the whole statistical physics. Therefore, the detailed investigations of conditions which lead to ergodicity breaking, and recognition of systems in which the ergodic hypothesis may cease to hold are important.

In the recent literature [1-4], the process $X(t)$ is called weakly nonergodic when

$$
\lim _{t_{f} \rightarrow \infty}\left\langle\overline{\delta^{2} X\left(t, t_{f}\right)}\right\rangle \neq\left\langle X^{2}(t)\right\rangle,
$$

(throughout this paper $X(t=0)=0)$ with

$$
\overline{\delta^{2} X\left(t, t_{f}\right)}=\frac{1}{t_{f}-t} \int_{0}^{t_{f}-t} d s[X(s+t)-X(s)]^{2}, \quad t \ll t_{f},
$$

* Presented at the XXV Marian Smoluchowski Symposium on Statistical Physics, "Fluctuation Relations in Nonequilibrium Regime", Kraków, Poland, September 10-13, 2012. 
relating two positions of the process (walker) separated by a time lag $t$ (for more details $c f$. [4] and references therein).

Weak ergodicity breaking (WEB) was discussed mainly in the context of anomalous diffusion (AD) [2], though recently it was shown that WEB is typical for much wider class of cumulative-growth processes, both stochastic and deterministic [4]. Experimentally, WEB was found in composite disordered systems: various glasses, cytoplasm of living cells, and like [3, 5], which seems to imply that AD and WEB are the properties of complicated structures.

It was shown in a recent paper [6] that anomalous diffusion (Brownian motion) can be generated and measured also in much simpler systems, easier to deal with and better suitable for various experimental manipulations. In this note, we want to discuss in detail the WEB in such systems.

\section{Time-dependent temperature}

Consider the Brownian motion (BM) in a medium with variable temperature [7]. The thermal (white Gaussian) noise $\xi(t)$ driving the Brownian particle is characterized by temperature- and density-dependent intensity

$$
\langle\xi(t)\rangle=0, \quad\langle\xi(t) \xi(s)\rangle=\sigma_{\xi}^{2}(T, \rho) \delta(t-s) .
$$

Let $\sigma_{\xi}^{2}$ be changing in time through $T=T(t)$, with $\rho=$ const. For an ideal medium $\sigma_{\xi}^{2} \sim T$ (fluctuation-dissipation theorem), and we may write $\sigma_{\xi}^{2}(T)=\left(T / T_{0}\right) \sigma_{0}^{2}\left(T_{0}\right)$, where $T_{0}>0$ is some reference temperature, e.g., $T(t=0)$; however, in real systems, such dependence can be more complicated, depending on details of interparticle interactions. In spatially nonuniform systems, the noise intensity will also depend on the (coarsegrained) position $\boldsymbol{r}, \sigma_{\xi}=\sigma_{\xi}(\boldsymbol{r}, t)$ (time-dependent diffusion coefficient was used recently for the description of random walks in porous materials [8]).

The Brownian motion driven by the noise with $\sigma_{\xi}=\sigma_{\xi}(t)$ is weakly nonergodic. Consider the process called scaled Brownian motion (SBM) ${ }_{\mathrm{s}} X_{\beta}(t)$ and defined by the Langevin equation [9]

$$
\frac{d}{d t}{ }^{s} X_{\beta}(t)=t^{\beta} \xi_{0}(t), \quad \beta>-1 / 2,
$$

with formal solution

$$
{ }_{\mathrm{s}} X_{\beta}(t)=\int_{0}^{t} u^{\beta} \xi_{0}(u) d u,
$$

where $\xi_{0}$ is the thermal noise at constant reference temperature $T_{0}$, with dispersion $\sigma_{0}^{2}\left(T_{0}\right)$. Define now the scaled thermal noise $\eta(t)=t^{\beta} \xi_{0}(t)$. Then,

$$
\langle\eta(t)\rangle=0, \quad\langle\eta(t) \eta(s)\rangle=\sigma_{\eta}^{2}(t) \delta(t-s), \quad \sigma_{\eta}(t)=t^{\beta} \sigma_{0},
$$


and

$$
\frac{d}{d t} \mathrm{~s} X_{\beta}(t)=\eta(t) \text {. }
$$

It was shown in [4] that SBM is weakly nonergodic. This proof can be easily extended for any Brownian motion driven by thermal noise $\xi(t)$ with time-dependent intensity $\sigma_{\xi}(t)=\phi(t) \sigma_{0}$ (in fact, this follows directly from the formulas (2.2)-(2.3) of [4]; for the sake of completeness, we present here the specialized calculations). Namely, for

$$
X(t)=\int_{0}^{t} d s \phi(s) \xi(s),
$$

we get from Eqs. (1), (2) and (8):

$$
\begin{aligned}
\left\langle X^{2}(t)\right\rangle & =2 \int_{0}^{t} d s D(s) \\
2 D(t) & =\sigma_{0}^{2} \phi^{2}(t)=\sigma_{\xi}^{2}(t, T(t)) \\
\left\langle\overline{\delta^{2} X\left(t, t_{f}\right)}\right\rangle & =\frac{2}{t_{f}-t} \int_{0}^{t_{f}-t} d s \int_{s}^{s+t} d u D(u) \\
& =\frac{1}{t_{f}-t} \int_{0}^{t_{f}-t} d s\left[\left\langle X^{2}(s+t)\right\rangle-\left\langle X^{2}(s)\right\rangle\right]
\end{aligned}
$$

which is weakly nonergodic except for $D=$ const., i.e., for the Wiener process (with constant temperature), and maybe for some special combinations of the spatial and temporal dependence of $D(\boldsymbol{r}, t)$ (i.e., $T(\boldsymbol{r}, t)-c f$. Eq. (27) below with $\gamma=-2 \beta$ for an example).

When the heating function $\phi(t)$ is proportional to $t^{\beta}$ (SBM case), this setup enables the realization of both WEB, and anomalous diffusion. Other forms of $\phi(t)$ will lead to non-diffusional (i.e., with dispersion not scaling algebraically with time) behaviour of the (weakly nonergodic) Brownian motions.

For example, let $\phi(t)=1+A \sin (\omega t), A^{2}<1$. This gives [6]

$$
\left\langle X^{2}(t)\right\rangle=2 D_{A} t+4 D_{0} \frac{A}{\omega}\left[1-\cos (\omega t)-\frac{A}{4} \sin (2 \omega t)\right],
$$

where $D_{A}=D_{0}\left(1+A^{2} / 2\right), 2 D_{0}=\sigma_{0}^{2}\left(T_{0}\right)$, which tends asymptotically to normal diffusion with higher effective diffusion coefficient (enhanced diffusion or accelerated Brownian motion) [6]. When this result is averaged over 
phase $\varphi(\omega t \rightarrow \omega t+\varphi)$, the oscillating part vanishes, but the enhancement remains. The same effect will appear for anomalous Brownian motions, and for other forms of driving $\phi(t)$, either periodic or random. Note that when the asymptotic temporal average of the changes of temperature is zero, as in Eq. (12), we have a kind of pumping of energy into the Brownian particle, analogous to e.g. ionic nanopumping in asymmetric nanopores [10].

The spatial analog of this situation could be realized experimentally in the so-called bistable ballast resistor [11].

Inserting Eq. (12) into Eq. (11), one gets

$$
\begin{aligned}
\left\langle\overline{\delta^{2} X\left(t, t_{f}\right)}\right\rangle= & 2 D_{A} t+\frac{4 D_{0} A^{2}}{\omega^{2}\left(t_{f}-t\right)}\left\{\sin \left[\omega\left(t_{f}-t\right)\right]-\sin \left(\omega t_{f}\right)\right\} \\
& -\frac{D_{0} A}{2 \omega^{2}\left(t_{f}-t\right)}\left\{\cos \left[2 \omega\left(t_{f}-t\right)\right]-\cos \left(2 \omega t_{f}\right)\right\} .
\end{aligned}
$$

Thus,

$$
\lim _{t_{f} \rightarrow \infty}\left\langle\overline{\delta^{2} X\left(t, t_{f}\right)}\right\rangle=\lim _{t \rightarrow \infty}\left\langle X^{2}(t)\right\rangle, \quad t \ll t_{f},
$$

i.e., the process Eq. (12) is asymptotically ergodic.

\section{Spatially nonuniform temperature}

When the heating/cooling of the system is spatially inhomogeneous, the position- and time-dependent temperature profile $T=T(\boldsymbol{r}, t)$ has to be calculated from the Fourier-Kirchhoff equation (FKE)

$$
\frac{\partial T}{\partial t}=\Lambda \frac{\partial^{2} T}{\partial \boldsymbol{r}^{2}}+S(\boldsymbol{r}, t)
$$

where $\Lambda$ is the heat conduction coefficient and $S$ - heat source. The analytic solution of this equation can be found for very few special cases. On the other hand, the FKE can be used for finding the space- and time-dependent heat flow $S(\boldsymbol{r}, t)$ necessary for the attaining the prescribed temperature profile $T=T(\boldsymbol{r}, t)$.

Analytic treatment of the Brownian motion with $\sigma_{\xi}(T(t, \boldsymbol{r}))$ is possible for a few special cases only (cf. [6]). Let $T(\boldsymbol{r}, t)=T_{0} \phi^{2}(t) \psi^{2}(\boldsymbol{r})$. Then,

$$
d X(t) / d t=\phi(t) \psi(\boldsymbol{r}) \xi_{0}(t) .
$$

Assume that the reaction of the Brownian particle on the stochastic "kicks" is retarded (Stratonovich interpretation of stochastic integrals [12]). Then, Eq. (16) can be written in the form

$$
d Y(t, \boldsymbol{r}) / d t=\phi(t) \xi_{0}(t), \quad d Y(t, \boldsymbol{r})=d X(t, \boldsymbol{r}) / \psi(\boldsymbol{r}) .
$$


Note that for stationary systems, $\phi=$ const., the process $\{Y(t)\}$ becomes the standard Wiener process $\{W(t)\}$.

The properties of the original process $\{X(t)\}$ can be obtained by the inversion of the transform (17), which again can be done analytically for some special cases only.

Consider, for simplicity, the one-dimensional case $\boldsymbol{r}=X$. The formal solution of Eq. (16) reads

$$
X(t)=\int_{0}^{t} d s \psi(X(s)) \phi(s) \xi(s) .
$$

Making use of the Stratonovich interpretation of stochastic integrals, we get

$$
\left\langle X^{2}(t)\right\rangle=2 D_{0} \int_{0}^{t} d s\left\langle\psi^{2}(X(s))\right\rangle \phi^{2}(s) .
$$

Analogous calculations of the time average, Eq. (2), give

$$
\begin{aligned}
\left\langle\overline{\delta^{2} X\left(t, t_{f}\right)}\right\rangle & =\frac{1}{t_{f}-t} \int_{0}^{t_{f}-t} d s\left\langle[X(s+t)-X(s)]^{2}\right\rangle \\
& =\frac{2 D_{0}}{t_{f}-t} \int_{0}^{t_{f}-t} d s \int_{s}^{s+t} d u\left\langle\psi^{2}(X(u))\right\rangle \phi^{2}(u) .
\end{aligned}
$$

Thus, even for the stationary case $\phi=1$, the process $\{X(t)\}$ is ergodic only when the second integral in Eq. $(20) \neq f(s)$, which is fulfilled for $\psi=$ const., but besides maybe only for some very special choices of the temperature gradients. This proves that in most realistic cases the processes $\{X(t)\}$ in the media with spatial temperature gradients are weakly nonergodic.

Further analysis depends on the forms of the functions $\phi(t)$ and $\psi(\boldsymbol{r})$.

Simplest and easiest for experimental realization spatially nonuniform system is the stationary sample (e.g. rod or fluid-filled cylinder) of length $L$, with isolated walls, kept at constant temperature $T(X=0)=T_{0}$ and $T(X=L)=T_{L}$, i.e., with stationary temperature gradient $T(X)=T_{0}+a X$, $a=\left(T_{L}-T_{0}\right) / L$. Then $S(\boldsymbol{r}, t)=0$ for $0<X<L$, and the Langevin equation reads

$$
\dot{X}(t)=\xi(t)=\left[T(X) / T_{0}\right] \xi_{0}(t)=(1+b X) \xi_{0}(t), \quad b=a / T_{0},
$$


i.e.,

$$
X(t)=\frac{1}{b}\left[e^{a W_{0}(t)}-1\right], \quad W_{0}(t)=\int_{0}^{t} d s \xi_{0}(s),
$$

$W_{0}(t)$ being the Wiener process driven by thermal noise $\xi_{0}(t)$ at temperature $T_{0}$, i.e., $\left\langle W^{2}(t)\right\rangle=2 D_{0} t$.

Thus [13],

$$
\left\langle X^{2}(t)\right\rangle=\frac{1}{b^{2}}\left\langle\left[e^{a W_{0}(t)}-1\right]\right\rangle=\frac{1}{b^{2}}\left(e^{4 a^{2} D_{0} t}-2 e^{a^{2} D_{0} t}+1\right) .
$$

The Wiener process $W_{0}(t)$ is ergodic, however,

$$
\begin{aligned}
\left\langle\overline{\delta^{2} X\left(t, t_{f}\right)}\right\rangle & =\frac{1}{t_{f}-t} \int_{0}^{t_{f}-t} d s[X(s+t)-X(s)]^{2} \\
& =\frac{1}{t_{f}-t} \int_{0}^{t_{f}-t} d s\left\langle e^{2 a W_{0}(s+t)}-2 e^{a W_{0}(s+t)} e^{a W_{0}(s)}+e^{a W_{0}(s)}\right\rangle \\
& =\left\langle X^{2}(t)\right\rangle \frac{e^{4 a^{2} D_{o}\left(t_{f}-t\right)}-1}{4 a^{2} D_{0}} .
\end{aligned}
$$

The latter results from the following

$$
\begin{aligned}
\left\langle e^{a W_{0}(s+t)} e^{a W_{0}(s)}\right\rangle & =\left\langle\exp \left\{2 a \int_{0}^{s} d z \xi_{0}(z)\right\} \exp \left\{a \int_{s}^{s+t} d z \xi_{0}(z)\right\}\right\rangle \\
& =\exp \left\{a^{2} D_{0}(4 s+t)\right\}
\end{aligned}
$$

from the fact that i.i.d. white thermal noises $\xi_{0}(t)$ at disjoint time intervals are uncorrelated, and from the properties of averages of Gaussian-distributed random series [13].

Thus, although the Wiener process $W_{0}(t)$ is ergodic, the process $X(T)$, Eq. (22) is weakly nonergodic.

Brownian motion in a more general nonstationary and nonuniform system with $(X \in[-L,+L], L \rightarrow \infty)$, initial condition $T(X=0, t=0)=$ $T_{0}=$ const., $X(t=0)=0$, and $T=T_{0} \phi^{2}(t) \psi^{2}(X)=T_{0}(t+\tau)^{2 \beta}(|X|+a)^{2 \gamma}$, $\tau>0, a>0$ was analyzed in [6]. In this case, the heating function and the solutions are

$$
\begin{aligned}
S(X, t) / T_{0} & =\psi^{2}(X) d \phi^{2}(t) / d t-\Lambda \phi^{2}(t) d^{2} \psi^{2}(X) d X^{2} \\
& =2(|X|+a)^{2 \gamma-2}(t+\tau)^{2 \beta-1}\left[(|X|+a)^{2}-\Lambda(t+\tau)\right],
\end{aligned}
$$




$$
\begin{aligned}
& Y(t)=\operatorname{sgn}(X(t)) \begin{cases}\mu(|X|+a)^{1-\gamma}, & \gamma \neq 1, \\
a \ln (|X(t)| / a+1), & \gamma=1,\end{cases} \\
& X(t)= \begin{cases}\operatorname{sgn}(Y(t) / \mu)\left[|Y(t) / \mu|^{\mu}-a\right], & \gamma \neq 1, \\
\operatorname{sgn}(X(t)) a\left[e^{Y(t) \operatorname{sgn}(X(t))}-1\right], & \gamma=1,\end{cases}
\end{aligned}
$$

where $\mu=1 /(1-\gamma)$, and $\operatorname{sgn}(f)$ denotes the sign of $f(\operatorname{sgn}(0)=0)$. Note that the initial condition $X(t=0)=0$ implies $Y(t=0)=0$. This gives the dispersions

$$
\left\langle Y^{2}(t)\right\rangle=2 D_{\beta}(t+\tau)^{\alpha_{0}}, \quad \alpha_{0}=2 \beta+1,
$$

and for $t_{f} \rightarrow \infty, t \ll t_{f}$

$$
\left\langle\overline{\delta^{2} Y\left(t, t_{f}\right)}\right\rangle \rightarrow 2 D_{\beta} t t_{f}^{\alpha_{0}-1},
$$

where $D_{\beta}=D_{0} / \alpha_{0}$. Thus [13], for $\gamma \neq 1$

$$
\begin{aligned}
\left\langle X^{2}(t)\right\rangle & =2 D_{2}(t+\tau)^{\alpha}-2 a D_{1}(t+\tau)^{\alpha / 2}+a^{2}, \\
\alpha & =\mu \alpha_{0}=(2 \beta+1) /(1-\gamma), \\
2 D_{2} & =\left(2 D_{\beta} / \mu^{2}\right)^{\mu} \Gamma(\mu+1 / 2) \sqrt{2 / \pi} \\
2 D_{1} & =\left(2 D_{\beta} / \mu^{2}\right)^{\mu / 2} \Gamma((\mu+1) / 2) \sqrt{2 / \pi},
\end{aligned}
$$

and for $\gamma=1$

$$
\left\langle X^{2}\right\rangle=a^{2}\left[\exp \left\{4 D_{\beta}(t+\tau)^{\alpha_{0}}\right\}-2 \exp \left\{D_{\beta}(t+\tau)^{\alpha_{0}}\right\}+1\right] .
$$

Note that $\gamma=1$ implies parabolic increase of temperature $T(X)$ with distance from the center. On the other hand, when $\beta+\gamma>1 / 2$, we get $\alpha>2$, i.e., superballistic Brownian motion. These results show that the Brownian motion accelerates exponentially in stationary systems with parabolic increase of temperature from the center towards the ends of a long (formally, infinitely long) cylinder. This means also that the logarithm of the dispersion of $X(t)$ behaves asymptotically as the normal diffusion. Linear $T(X)=T_{0}(|X|+a)$ results in the parabolic acceleration of the Brownian motion (ballistic motion).

Direct calculations of $\left.\overline{\delta^{2} X\left(t, t_{f}\right)}\right\rangle$ cannot be done analytically [14]. However, to prove that the original process $\{X(t)\}$ is weakly nonergodic, it is sufficient to prove that the ensemble and time averages of any function of this process are not equal. Therefore, the result (25)-(26) for the averages of $Y(t)=f(X(T))$ proves the weak ergodicity breaking of diffusional 
processes in systems with spatially nonuniform and/or time-dependent temperature $T(t, x)$. Note, however, that for $\gamma=-2 \beta$ we get $\alpha=1$, i.e., the process $\{X(t)\}$ becomes the normal ergodic diffusion.

The results discussed in this paper show that the anomalous nonergodic transport is not restricted to composite systems, but can be easily generated in almost any body. Therefore, by (uniform) heating of the system with changes of temperature proportional to $t^{\beta}$, one can measure directly the weakly-nonergodic anomalous diffusion (both subdiffusion for $-1 / 2<$ $\beta<0$, slowing-down the heating, and superdiffusion for $0<\beta<1 / 2$, accelerating the heating).

A corollary to this implies that the Mandelbrot-Van Ness (cf. [4]) fractional Brownian motion $B_{H}(t)$ which is ergodic, will become weakly nonergodic in systems with varying temperature. Viz., the MBM of [4] can be written as $B_{H}$ driven by $\eta(t)$, Eq. (6)

$$
\begin{aligned}
B_{H}(t)= & \frac{1}{\Gamma(H+1 / 2)}\left[\int_{-\infty}^{t}(t-u)^{H-1 / 2} \eta(t ; T(t)) d t\right. \\
& \left.-\int_{-\infty}^{0}(-u)^{H-1 / 2} \eta(t ; T(t)) d t\right] \\
= & \frac{1}{\Gamma(H+1 / 2)}\left[\int_{-\infty}^{t}(t-u)^{H-1 / 2} \phi(u) d W(u)\right. \\
& \left.-\int_{-\infty}^{0}(-u)^{H-1 / 2} \phi(u) d W(u)\right]
\end{aligned}
$$

where $d W(t)=\xi\left(t, T_{0}\right) d t$, and $W(t)$ is the Wiener process at temperature $T_{0}$.

\section{Macroscopic diffusion}

Macroscopic diffusion is described by the Ficks' law

$$
\frac{\partial c(\boldsymbol{r}, t)}{\partial t}=\nabla\{D(\boldsymbol{r}, t) \nabla c(\boldsymbol{r}, t)\},
$$

where $c(\boldsymbol{r}, t)$ is the concentration of the diffusing substance, and $D(\boldsymbol{r}, t)=$ $D_{\text {macro }}$ is the macroscopic diffusion coefficient. For diluted ideal systems, $D_{\text {macro }}$ can be identified with the used above $D=D_{\text {micro }}$ being the microscopic diffusion coefficient for corresponding Brownian motion which implies that $D_{\text {macro }}(T) \sim T$. Such identification can be done "because the process 
of diffusion is the result of superposition of Brownian motions of different molecules of diffusing substance" [15]. For dense real systems, $D_{\text {macro will }}$ depend not only on temperature and density, but also on the concentrations of all species constituting the considered system.

For spatially uniform systems, when $D=D(T(t))=\phi(t) D_{0}$ and $D$ does not depend on concentration, the substitution $d \tau=\phi(t) d t$ implies [16] that the shape of spatial concentration profile $c\left(\boldsymbol{r}, t ; D(T(t))=c\left(\boldsymbol{r}, \tau ; D_{0}\right)\right.$ remains unchanged, with the rate of establishing this profile depending now on $\tau$ instead of $t$.

When $D$ depends on time $t$ and position $\boldsymbol{r}$ through its dependence on concentration $c$ only, i.e., $D=D(\boldsymbol{r}, t ; c)=D(c(\boldsymbol{r}, t))$, the analytic solutions can be found (for some cases) by the Boltzmann transform $\boldsymbol{y}=\boldsymbol{r} \sqrt{t} / 2$, which gives $D(c(\boldsymbol{r}, t))=\chi(\boldsymbol{y}) D_{0}(c)$ (for details $c f$. [16]). In this case, Eq. (30) reads

$$
-2 \boldsymbol{y} \cdot \nabla \boldsymbol{y} c(\boldsymbol{y})=\nabla \boldsymbol{y}\left\{D_{0}(c) \chi(\boldsymbol{y}) \nabla \boldsymbol{y} c(\boldsymbol{y})\right\}
$$

\section{Final remarks and conclusions}

It was shown here that weak ergodicity breaking can be detected in simple systems with nonstationary or/and nonuniform temperature. One may expect that similar effects should appear in systems driven by external forces [17], and in systems with changing density (the latter through the density dependence of the diffusion coefficients), either in inflationary spaces (spatially uniform systems) or in expanding/contracting gases (spatially nonuniform systems).

The results and assertions described in this paper (and in [6]) can be checked experimentally. This can be done by standard measurements of the time-dependence of the dispersion $\left\langle X^{2}(t)\right\rangle$ and - when possible — by the measurements of the single trajectories of Brownian particles. The latter would give direct experimental verification of nonergodic character of SBM, and of other processes of this type. Note that the same can be done with other time characteristics of the speed of heating/cooling, including natural exponential cooling by radiation. It is important (and obvious) in such measurements that the time-scales of heating/cooling and of diffusion should be of the same order of magnitude. Note that when the cooling/heating is spatially uniform, there will appear no temperature gradients through the system, and we shall get the system in a quasi-adiabatically shifting thermal equilibrium.

Nonuniform heating can be realized in almost any system, by almost any means. However, one should remember that in such systems the temperature gradients $\nabla T$ lead to appearance of thermoosmotic flows caused 
by additional concentration gradients $(\nabla c)_{T}$ (Ludwig-Soret effect $\left.[18,19]\right)$

$$
(\nabla c)_{T}=-S_{T} c_{0}\left(1-c_{0}\right) \nabla T,
$$

where $c=c(X)$ is the particle number (mole) fraction of the diffusing species (i.e., traced Brownian particle), $c_{0}$ - its equilibrium value, and $S_{T}$ - the so-called Soret coefficient. The contamination of measured diffusional flows by this effect can be reduced when either $S_{T}$ or $c_{0}$ are small enough. On the other hand, thermoosmotic flows can be subtracted from measured diffusional flows - the values of the Soret coefficients are known for simple mixtures and solutions.

No thermoosmotic flows will appear in systems with spatially uniform, changing in time temperature. In this case, experimental realizations of bulk uniform heating were discussed in [6]. For the sake of completeness, let us repeat: (i) heating of solutions in water (or in other dielectric solvents and their mixtures containing -OH groups - fats, alcohols, and like) can be realized in microwave ovens; (ii) other options which also can be easily realized experimentally are (quasi) one-and two-dimensional systems: monomolecular films on heated/cooled support, and thin flat layers or long thin rods or fluid-filled cylinders, cooling or heating e.g. by emitting or absorbing radiation, or immersed in a heat bath.

As we have mentioned, the analytic treatment of the Brownian motion in systems with spatial temperature gradients is in most cases very difficult (or just impossible). Even for the realistic situation: constant in time point heating, i.e., for

$$
S(\boldsymbol{r}, t)=\left\{\begin{array}{l}
A T^{*}, \boldsymbol{r}=0, \forall t, T^{*}=\text { const. } \\
0, \boldsymbol{r} \neq 0, \forall t
\end{array}\right.
$$

when the temperature profile resulting from the FKE is Gaussian (for $\boldsymbol{r} \neq 0$ ) the analytic inversion of the transform (17) cannot be done. Nevertheless, one may expect that the behaviour of the Brownian particles in such simple experimentally arrangements should be similar, at least in a qualitative fashion, to the cases discussed in this paper. On the other hand, although the analytic calculations of dispersions (both ensemble and temporal) are too difficult, it is possible to find the averages of some functions of $X(t), e . g$. the inversions $Y(t)$ of the process $X(t)$, or some functions of these inversions. The ergodicity-breaking by the transformed process $Y(t)$ will be sufficient to prove the ergodicity-breaking by the original process $X(t)$, however, the ergodic behaviour of $Y(t)$ does not imply the same for $X(t)$. 


\section{REFERENCES}

[1] A. Fuliński, E. Gudowska-Nowak, Acta Phys. Pol. B 22, 457 (1991).

[2] G. Bel, E. Barkai, Phys. Rev. Lett. 94, 240602 (2005); H. Tanaka, S. Jabbari-Farouji, J. Meunier, D. Bonn, Phys. Rev. E71, 021402 (2005);

L. Cipeletti, L. Ramos, J. Phys.: Condens. Matter 17, R253 (2005); Y. He, S. Burov, R. Metzler, E. Barkai, Phys. Rev. Lett. 101, 058101 (2008); A. Lubelski, I.M. Sokolov, J. Klafter, Phys. Rev. Lett. 100, 250602 (2008); M. Magdziarz, A. Weron, K. Burnecki, J. Klafter, Phys. Rev. Lett. 103, 180602 (2009); V. Tejedor, R. Metzler, J. Phys. A43, 082002 (2010); S. Burov, J.H. Jeon, R. Metzler, E. Barkai, Phys. Chem. Chem. Phys. 13, 1800 (2011); M. Magdziarz, A. Weron, Phys. Rev. E84, 051138 (2011).

[3] V. Tejedor et al., Biophys. J. 98, 1364 (2010); A.V. Weigel, B. Simon, M.M. Tamkun, D. Krapf, PNAS 108, 6438 (2011); E. Kepten, I. Bronshtein, Y. Garini, Phys. Rev. E83, 041919 (2011); J.-H. Jeon et al., Phys. Rev. Lett. 106, 048103 (2011); L. Oddershede, R. Metzler, Phys. Rev. Lett. 106, 048103 (2011).

[4] A. Fuliński, Phys. Rev. E83, 061140 (2011).

[5] J.P. Bouchaud, J. Phys. I France 2, 1705 (1992); I.Y. Wong et al., Phys. Rev. Lett. 92, 178101 (2004); L. Cipeletti, L. Ramos, J. Phys.: Condens. Matter 17, R253 (2005); H. Tanaka, S. Jabbari-Farouji, J. Meunier, D. Bonn, Phys. Rev. E71, 021402 (2005); J. Szymanski, M. Weiss, Phys. Rev. Lett. 103, 038102 (2009); M. Magdziarz, A. Weron, K. Burnecki, J. Klafter, Phys. Rev. Lett. 103, 180602 (2009); M. Magdziarz, A. Weron, Phys. Rev. E84, 051138 (2011).

[6] A. Fuliński, J. Chem. Phys. 138, 021101 (2013) [arXiv:1206.4594 [cond-mat.stat-mech]].

[7] We consider here the heating/cooling of the medium in which the Brownian particle moves. This is different from the situation when it is the Brownian particle itself which is heated - cf. L. Joly, S. Merabia, J.-L. Barrat, Europhys. Lett. 94, 50007 (2011); D. Chakraborty et al., 96, 60009 (2011) and references therein.

[8] Z. Zhang, D.L. Johnson, L.M. Schwartz, Phys. Rev. E84, 031129 (2011).

[9] S.C. Lim, S.V. Muniandy, Phys. Rev. E66, 021114 (2002); cf. also

K.G. Wang, M. Tokuyama, Physica A 265, 341 (1999).

[10] Z. Siwy, A. Fuliński, Phys. Rev. Lett. 89, 198103 (2002).

[11] B. Ross, J.D. Litster, Phys. Rev. A15, 1246 (1977); R. Landauer, Phys. Rev. A15, 2117 (1977).

[12] Cf. W. Horsthemke, R. Lefever, Noise-Induced Transitions, Springer, Berlin 1984; J.M. Sancho, Phys. Rev. E64, 062102 (2011) and references therein.

[13] $\{Y(t)\}$ is Gaussian-distributed; threfore, $\langle\exp ( \pm Y)\rangle=\exp \left(\left\langle Y^{2}\right\rangle / 2\right)$, and $\left\langle|Y|^{\nu}\right\rangle=\left(2\left\langle Y^{2}\right\rangle^{\nu} / \pi\right)^{1 / 2} \Gamma((\nu+1) / 2)$. 
[14] The comparison of the results for $\left\langle X^{2}(t)\right\rangle$ and $\left\langle Y^{2}(t)\right\rangle$ seems to suggest that also the average $\left\langle\overline{\left\langle\delta^{2} X\left(t, t_{f}\right)\right.}\right\rangle$ should behave similarly as $\left\langle\overline{\delta^{2} Y\left(t, t_{f}\right)}\right\rangle$, i.e., that it should scale as $(t+\tau)\left(t_{f}+\tau\right)^{\alpha-1}$.

[15] M. Smoluchowski, Ann. Phys. 48, 1103 (1915).

[16] J. Crank, The Mathematics of Diffusion, II-nd Ed., Sect. 7.1, Clarendon Press, Oxford 1975.

[17] M.A. Despósito, Phys. Rev. E84, 061114 (2011).

[18] H. Cabrera et al., J. Chem. Phys. 131, 031106 (2009).

[19] R. Kita, S. Wiegand, J. Luettner-Strathmann, J. Chem. Phys. 121, 3874 (2004). 